\title{
Automated Topology Design for Electromagnetic Devices
}

\author{
Gullu Kiziltas ${ }^{1,2}$, John L. Volakis ${ }^{1,2}$ and Noboru Kikuchi ${ }^{1}$ \\ ${ }^{(1)}$ The University of Michigan, Ann Arbor, MI 48109-2122 \\ ${ }^{(2)}$ ElectroScience Lab., ECE Dept., The Ohio State University, Columbus, OH 43212-1191
}

\begin{abstract}
In this paper a novel automated design procedure based on the integration of a full wave Finite Element Analysis (FEA) and a topology design method employing Sequential Linear Programming (SLP) is introduced. The employed topology design method is the Solid Isotropic Material with Penalization (SIMP) technique which is formulated as a general non-linear optimization problem. SLP is used to solve the optimization problem with the sensitivity analysis based on the adjoint variable method for complex variables. A key aspect of the proposed design method is the integration of optimization tools with a fast simulator based on the Finite Element-Boundary Integral (FEBI) method. The capability of the proposed design method is demonstrated by designing a patch antenna subject to prespecified bandwidth and miniaturization criteria. Results show that the proposed design method is capable of designing full three-dimensional volumetric material textures and printed conductor topologies for filters and patch antennas with enhanced performance.
\end{abstract}

\section{INTRODUCTION}

Design optimization for electromagnetic applications has traditionally been a process based on the creativity, past experience and knowledge of the designer. It has thus focused mostly on shape or geometry design of the device for performance improvement [1-3]. A more general design method is therefore likely to yield better overall performance. Such an approach draws from a broader class of design solutions as compared to conventional design methods and is capable of achieving designs that may yield much higher performance. Recent advances in fast and rigorous full wave simulators and the concurrent availability of inexpensive manufacturing techniques for intricate shape and composite materials provides the opportunity to revolutionize traditional design optimization processes to topology and material optimization $[4,5]$. This is expected to generate "new" electromagnetic devices that exhibit new properties and significantly better performance. So far, there are very few examples in the literature on topology optimization of electrical devices [6-9] and the majority of these dealt with problem specific, restricted or semi-analytic tools for magneto-static applications. That is, the subject of topology and material optimization is a fresh approach for developing novel RF device designs.
Two critical issues have to be addressed in developing novel designs. First, we need a general mathematical framework to conduct rigorous analysis of composite materials without imposing any geometric and material restrictions. Second, a versatile design method is required to find the best geometric configuration and material composition of the device. In this paper, the Solid Isotropic Material with Penalization Method (SIMP) [8] is integrated with fast hybrid finite-element boundary integral simulations [9] to develop full three-dimensional antenna designs addressing these two limitations.

It is well known that high contrast substrates, although suitable for miniaturization lead to smaller bandwidth $[10,11]$. To overcome this limitation, the proposed design method extending the SIMP technique is employed to develop a miniature patch antenna with pre-specified bandwidth performance. SIMP incorporates a general non-linear optimization formulation based on well-defined optimization algorithms such as Sequential Linear Programming (SLP) [12] and employs a simple continuous function to relate the actual material property to the introduced "density" variable. As part of the solution technique, the adjoint sensitivity method [6] is employed to update the design variable in the optimization routine. This permits full interface with the FE-BI electromagnetic solver [9] and enhances the program

\section{CP712, Materials Processing and Design: Modeling, Simulation and Applications, NUMIFORM 2004,}

edited by S. Ghosh, J. C. Castro, and J. K. Lee

(C) 2004 American Institute of Physics 0-7354-0188-8/04/\$22.00 
efficiency. Pursuing the proposed design method and the inhomogeneous material modeling capability, we achieved $250 \%$ bandwidth improvement for a fixed size patch antenna on a high contrast substrate having a dielectric constant of $\varepsilon_{\mathrm{r}}=100$. Measurements were also carried out to validate the antenna's return loss performance.

The organization of the paper is as follows. First, the EM analysis tool is briefly introduced and subsequently the design procedure is outlined. This section starts with a discussion on the SIMP method and continues with the definition of the optimization model and its solution procedure. To demonstrate the capability of the proposed method, a 3D material design example for patch antenna radiation is tackled to achieve $250 \%$ bandwidth improvement over the traditional substrates.

\section{EM ANALYSIS}

As is the case with all numerical design optimization loops, the design process must incorporate a flexible and fast analysis module. It is also of crucial importance that this module be fast without compromising the generality of the geometry and material properties of the device.

For our case, a finite element (FE) simulator based on the well-established hybrid finite element-boundary integral (FE-BI) method is employed [9]. A periodic version of the FE-BI method is employed as described in [9] to provide for additional speed by using only a few modes and the FFT to carry out the matrix vector products. However, the optimization can be applied with any other analysis tool and for different applications other than printed antennas and/or arrays. As described in [9], the overall analysis method has $\mathrm{O}(\mathrm{N})$ memory demand and CPU complexity. By virtue of the finite-element method, the simulator is suitable for complex structures such as those involving inhomogeneous dielectrics, resistive patches, conducting patches and blocks, feed probes, impedance loads, etc. Accurate results have already been obtained for scattering and radiation by cavities, slots, and multilayer patch antennas and frequency selective surfaces, demonstrating the method's capability. The conventional implementation of the hybrid FE/BI method for doubly periodic arrays leads to a linear algebraic system of the following form:

$$
[A]\{E\}+[Z]\{E\}=\{f\}
$$

The A matrix is sparse and is associated with the FE portion of the hybrid method. Contributions of dielectric blocks or volumes and resistive cards or metallic edges in the unit cell are embedded in the [A] matrix. The [Z] matrix is associated with the edges on the top and bottom surfaces of the discretized unit cell and is fully populated. The right hand side vector $\{\mathrm{f}\}$ contains, as usual, excitations in the FE volume or BI apertures.

\section{DESIGN PROCEDURE}

Topology optimization methods are general design methods used to obtain simultaneously the best geometric and topological configuration in terms of geometry, physical dimensions, connectivity of boundaries and material implants. They have reached a level of maturity and are being applied successfully to many industrial problems for almost 20 years [13]. Recently, some applications have also appeared in the electromagnetics, the majority of which are restricted to specific magneto-static applications [6, 7]. For our design problem, we employ the topology optimization method based on the SIMP method to design miniature patch antennas with broadband behavior. The proposed method is aimed at designing the inhomogeneous structure of the dielectric substrate on which the patch is printed. Patch/conductor shape can also be used for further bandwidth improvements but is not considered here since the focus is on material design.

SIMP is very attractive to the engineering community because of its simplicity and efficiency. It basically synthesizes the device starting from any arbitrary topology. A key aspect of the design method is that any device, not known a priori, is represented by specifying the material properties at every point of the fixed design domain. For electromagnetic applications, these are the permittivity and permeability of the dielectric material and conductivity/resistance of the metallic patches, etc. [14]. In practice, to specify the material properties in the design region, the design space is discretized into material cells/finite elements. Actually, the most straightforward image-based geometry representation is the " $0 / 1$ " integer choice, where the design domain is represented by either a void or a filled/solid material and this was adopted in [3]. However, this formulation is not well-posed mathematically [8]. It can be wellposed by allowing for the design of materials with intermediate properties; that is, materials having graded properties. This is the essence of the SIMP method in which material grading is achieved by introducing a single density variable, $\rho$, and relating it to the actual material property of each finite element thru a continuous functional relationship. A suitable interpolation for the permittivity (and possibly a 
similar one for the resistance of a metallic patch would be):

$$
\rho=\left(\varepsilon_{\text {int }}-\varepsilon_{\text {air }}\right) /\left(\varepsilon_{\text {orig }}-\varepsilon_{\text {air }}\right)^{1 / n}
$$

where $\mathrm{n}$ is a penalization factor; $\varepsilon_{\text {int }}$ and $\varepsilon_{\text {orig }}$ are intermediate and original solid material permittivity, respectively. As $\mathrm{n}$ increases, intermediate values for the permittivity are less likely to occur, hence the term penalization for intermediate material. The on/off nature of the problem has been avoided through the introduction of the normalized density with $\rho=0$ corresponding to a void (air with $\varepsilon_{\text {air }}$ ), $\rho=1$ to solid (original material $\varepsilon_{\text {orig }}$ ) and $0<\rho<1$ to a graded intermediate dielectric material $\left(\varepsilon_{\text {int }}\right)$. Moreover, this parameterization allows for the formulation of the problem in a general non-linear optimization framework. The goal is to arrive at the optimum distribution of material (densities) such that a certain performance merit of a device is optimized subject to certain design constraints. The problem formulation and its solution will be discussed in the next section.

\section{SOLUTION OF THE OPTIMIZATION PROBLEM}

For our design problem, the specific goal is to determine the substrate material distribution under a square patch antenna subject to pre-specified bandwidth and miniaturization requirements. An appropriate model for the corresponding topology optimization problem employing the SIMP method would be to find the design variables $\rho$ that minimizes the cost function:

$$
f(\rho)=\min \left[\max \left(\left|s_{11}\right|_{j}\right)\right] j=1, \ldots N_{\text {freq }}
$$

subject to a material volume constraint:

$$
\sum_{i=1}^{N F E} \rho_{i} \cdot V_{i} \leq V^{*}
$$

and side constraints $(0 / 1)$ for each density variable.

Minimization of the highest return loss among sampled frequency points $\mathrm{N}_{\text {freq }}$ is known to maximize the return loss (S11) bandwidth [1] and the volume constraint basically limits the available amount of material. The design problem is easily recognized as a general non-linear optimization problem with usually several thousand variables/FEs. This makes the use of gradient-based optimization techniques a must for the optimization process. Due to its well-known efficiency and reliability, we specifically chose the Sequential Linear Programming (SLP) method employing the DSPLP package in the SLATEC library [15]. The essence of the SLP routine is to replace the objective function and constraints by their linear approximations at each iteration. Updates of the design variables are pursued thru the use of gradient information obtained thru the adjoint variable method [6], an efficient method that permits full interface with the FE-BI electromagnetic solver. We remark that this is an exact analysis based on the solution of the adjoint problem.

The sensitivity analysis is a crucial part of the design loop since it allows the integration of the solver with the SLP optimizer. The most critical aspect in doing so is to employ the gradients or the derivatives of the mathematical functions in the optimization model with respect to the design variables. Details on the sensitivity analysis are given in [6] and [16]. The linear programming sub-problem is then posed to find the optimal design changes from the current design point. It is of great importance to impose constraints for the design changes known as move limit bounds to ensure convergence. Typically, during one iteration, the design variables are allowed to change by $5-15 \%$ of their original values.

\section{THE DESIGN ALGORITHM}

The algorithm for the proposed design cycle is shown in Fig. 1. The design cycle starts with the initialization of the design variables, which corresponds to an initial homogenous dielectric substrate with a certain permittivity value. Certain design parameters are also specified at this step but do not change during the design cycle. These are design parameters such as patch geometry and material characterization, like the dielectric block dimensions. Also, the feed location, its amplitude and the frequency range of operation are specified. The next step is the discretization of the design domain into a large number of finite elements and the distribution of the available dielectric material throughout the domain. The iterations continue until convergence is achieved. To summarize, the following steps are executed as displayed: 1) Simulation of the device performance using the FE-BI solver and fed in data 2) Solution of the adjoint system equations of the original problem for the sensitivity analysis 3) Optimization of the material distribution of the dielectric material within the design domain using an SLP algorithm and 4) Updating of the design variables (densities/permittivities of design cells) relying on the interpolation scheme of the SIMP design method. Convergence is achieved when changes in the objective function value (hence changes in the design variables) drop below a certain value like $10^{-3}$. 


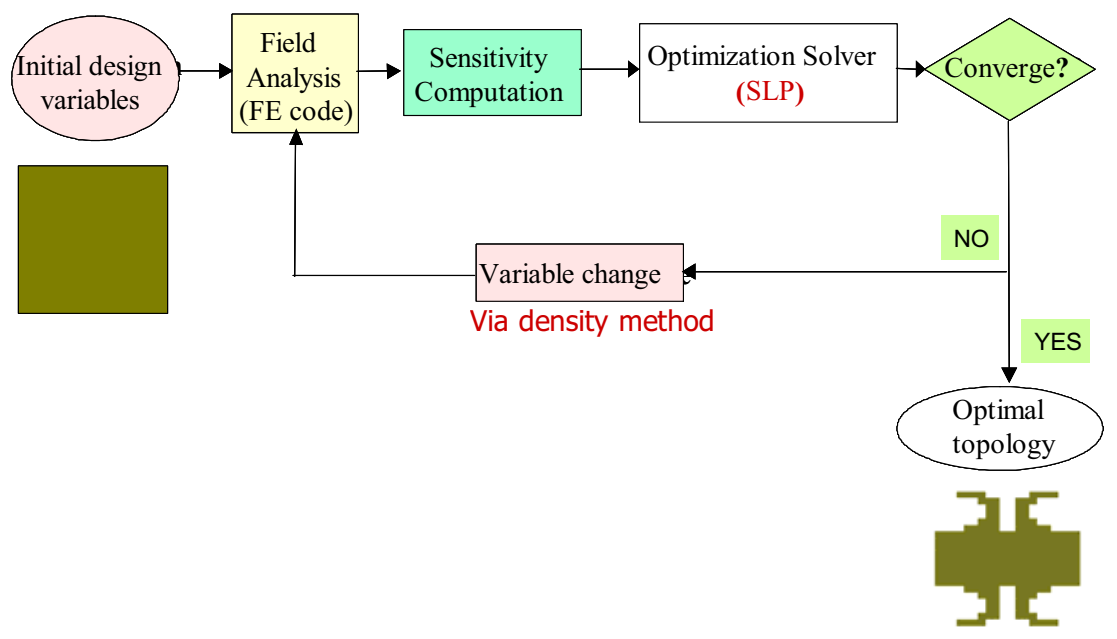

FIGURE 1. Design Optimization Flowchart.

\section{DESIGN EXAMPLE}

As is well known, microstrip patch antennas are attractive, low- weight, low-profile antennas which however suffer from low bandwidth. Moreover, its bandwidth is further reduced as the substrate dielectric constant is increased for miniaturization. In this section, we demonstrate the capability of the outlined design method to develop a small patch antenna subject to pre-specified bandwidth criteria. The main goal is to improve the bandwidth performance of a chosen simple patch antenna by introducing a new substrate texture (metamaterial) whose properties are not found in nature.

The details of the whole structure are displayed in Fig. 2. An initial homogenous substrate with $\varepsilon=42$ is chosen for the frequency range of interest $(1-2 \mathrm{GHz}$ sampled over 21 frequency points). The volume constraint is set to $70 \%$ to avoid trivial bandwidth improvement by a smaller dielectric constant. With each design cell being updated via the SIMP method and the SLP routine as described above, a graded volumetric design is obtained in 20 iterations. The computation time for the entire design process with 4000 finite elements and 21 frequency points per iteration, was 17 hours on a Pentium 3 Processor. The converged material distribution is displayed in Fig. 3 as a 3D color-coded block with each color pixel corresponding to a certain permittivity value (density value in scale bar). The corresponding return loss behaviors of the optimized and initial dielectric substrates are compared in Fig. 4. Given the poor bandwidth at the starting point of the design, i.e. a $5 \mathrm{~dB}$ return loss bandwidth of $6.7 \%$, the bandwidth performance obtained by designing for the material only, is truly remarkable. We were not able to obtain the typical $10 \mathrm{~dB}$ bandwidth for the initial design because of the capacitive nature of the high contrast material.

To fabricate the optimized design, certain post processing/image processing is needed to transform the $3 \mathrm{D}$ gray scale texture into a two-material solid composite. Adaptive Image Processing with a simple filtering idea based on a cut-off value of 0.64 for the densities has been adapted to solidify and fabricate the 3D material composite substrate as shown in Fig. 5, using Thermoplastic Green Machining [17]. More specifically, first a thermoplastic compound was prepared by mixing commercially available LowTemperature Cofirable Ceramic (LTCC- ULF 101) powder with melted binder systems. Once compounded, it was warm-pressed and the dielectric block in its "green body" state was obtained. The material is machinable at this stage and has slightly larger dimensions than the original design. After removing material via a computer-controlled equipment (Modela; Roland DG Corp., Japan) according to the filtered design geometry, the substrate is sintered. During machining, it is also slightly modified for machinability and considering shrinkage after sintering. In addition, to attain a smooth surface, the graded hole is filled with a polymer having a dielectric constant of 3 as depicted by the transparent material in Fig. 5. The return loss behavior of the fabricated design is compared in Fig. 4 for the initial 
substrate and the volumetric graded design. It is observed that the attained BW of the designed antenna has slightly changed after undergoing solidification steps described above. It is noted that the performance could have changed more drastically since the design is altered during the solidification process but it still represents a significant improvement over the initial design. This demonstrates the power of integrating robust optimization techniques with simple filtering processes for manufacturable substrates with improved performance.

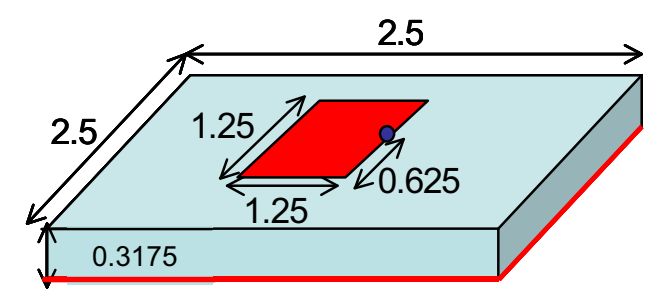

FIGURE 2. Schematic of patch antenna on initial homogeneous substrate (Dimensions are in $\mathrm{cm}$ ).

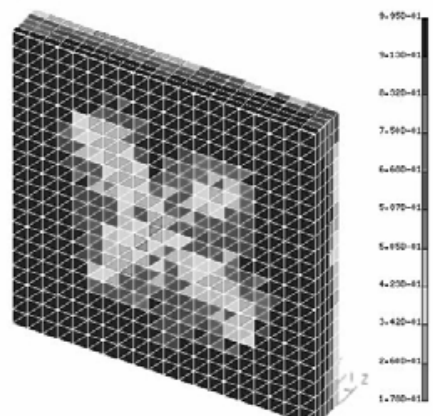

FIGURE 3. Optimized graded metamaterial substrate.

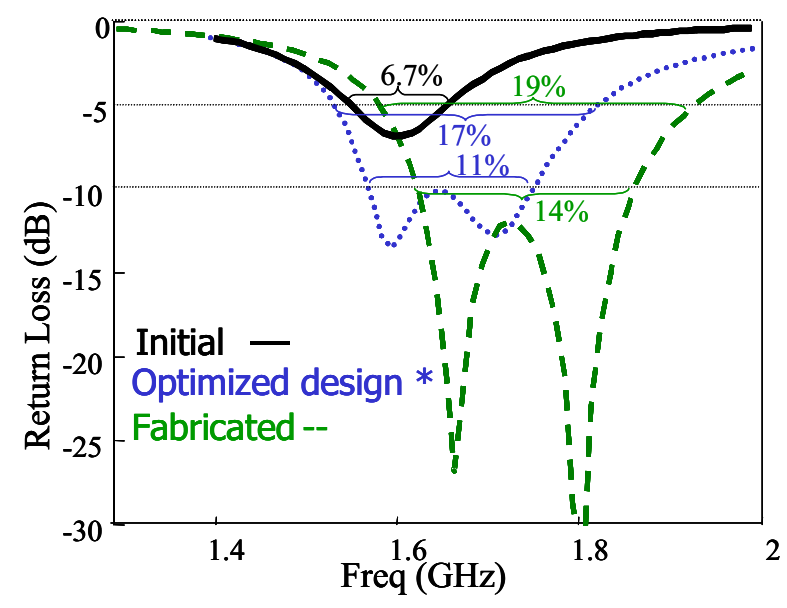

FIGURE 4. Return loss behaviour for initial, optimized and fabricated substrates.
To validate the performance of the metamaterial substrate, a square $1.25 \mathrm{~cm} \times 1.25 \mathrm{~cm}$ metallic patch is painted on top of the fabricated composite substrate using ECCOCOAT ${ }^{\circledR}$ C-110-5 silver paint. A coaxial probe feed is then used to excite the patch with geometrical details as shown in Fig. 2. The measurement results for the return loss performance of the metamaterial substrate are depicted in Fig. 6. It is noted that the bandwidth performance and its nulls of the fabricated metamaterial substrate agree very well in both the simulations and measurements with a frequency shift of $100 \mathrm{MHz}$. This small shift is attributed to the feed location and exact patch location uncertainties.

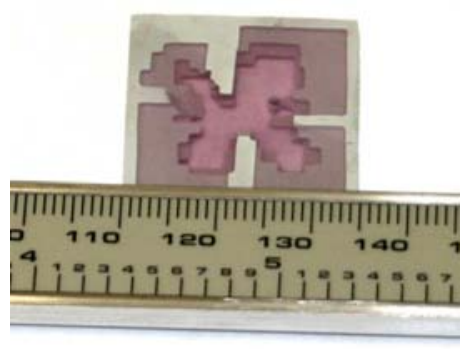

FIGURE 5. Fabricated two-material (LTCC with $\varepsilon=100$ filled with polymer of $\varepsilon=3$ ) composite substrate.

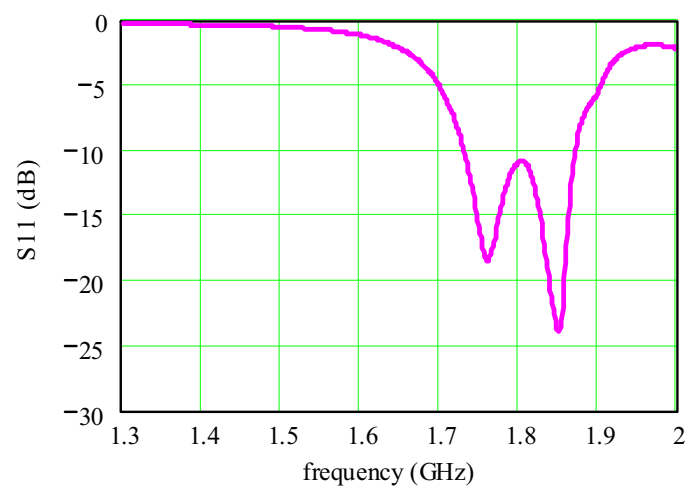

FIGURE 6. Measured return loss behaviour for fabricated metamaterial substrate (Fig. 5) with a probe fed patch antenna (Fig. 1).

\section{CONCLUSIONS}

A novel design procedure based on the integration of a full wave FEA and the SIMP design method employing SLP for the optimization is introduced. The capability is demonstrated by achieving a $250 \%$ BW improvement for a probe fed patch antenna by 
designing for its dielectric substrate subject to a constant size. The design is filtered and solidified to achieve a manufacturable design. The return loss response of the metamaterial is verified via measurements. The fabricated final design using TGM demonstrates the ability to design and manufacture novel material composites that have new properties never realized before. As demonstrated by the design example, by virtue of the generality and efficiency of the proposed method, there is great potential for improving antenna performance or other RF devices.

\section{ACKNOWLEDGMENTS}

The authors would like to acknowledge the material characterization and fabrication efforts by Angela Knapp and Dr. Younghag Koh under the supervision of Prof. Dr. John Halloran at the Material Science and Engineering Department at the University of Michigan. This study is supported by DARPA through the Naval Research Lab Grant N00173-01-1G910.

\section{REFERENCES}

[1] J.M. Johnson and Y. Rahmat-Samii, "Genetic algorithms and method of moments (GA/MoM) for the design of integrated antennas," IEEE Transactions on Antennas and Propagation, vol. 47, no. 10, pp. 1606-1614, October 1999

[2] E. Michielssen, J.M. Sajer, S. Ranjithan, and R. Mittra, "Design of light-weight, broad-band microwave absorbers using genetic algorithms," IEEE Transactions on. Microwave Theory and Techniques, vol. 41, no. 6/7, pp. 1024-1031, 1993.

[3] Z. Li, Y. E. Erdemli, J.L. Volakis, and P.Y. Papalambros, "Design Optimization of Conformal Antennas by Integrating Stochastic Algorithms With the Hybrid Finite-Element Method ," IEEE Transactions on Antennas and Propagation, vol. 50, no. 5, May 2002

[4] M.P. Bendsøe and N. Kikuchi, "Generating optimal topologies in structural design using a homogenization method," Computer Methods in Applied Mechanics and Engineering, 71:197--224, 1988

[5] M.P. Bendsøe, Methods for the Optimization of Structural Topology, Shape and Material, New York: Springer, 1989.

[6] D. N. Dyck, D.A. Lowther and E.M. Freeman, "A method of Computing the Sensitivity in Electromagnetic Quantities to changes in Materials and Sources," IEEE Transactions on Magnetics, vol. 30, no. 5, Sept. 1994
[7] J. Yoo and N. Kikuchi, "Topology Optimization in magnetic fields using the homogenization design method," International Journal for Numerical Methods in Engineering, vol. 48, pp. 1463-1479, 2000

[8] O. Sigmund, "Design of multi-physics actuators using topology optimization- Part I: One material structures", Computer Methods in Applied Mechanics and Engineering, vol. 190, pp. 65776604, 2001

[9] T. F. Eibert and J. L. Volakis, "Fast spectral domain algorithm for hybrid finite element / boundary integral modeling of doubly periodic structures," IEEE Proceedings: Microwaves, Antennas and Propagation, vol. 147, no. 5, pp. 329-334, October 2000

[10]R.C. Hansen, "Fundamental limitations on antennas," Proceedings of IEEE, vol. 69, pp. 170$182,1981$.

[11] T. K. Lo, C. O. Ho, Y. Hwang, E. K. W. Lam, and B. Lee, "Miniature aperture-coupled microstrip antenna of very high permittivity," Electronics Letters, vol. 33, pp. 9-10, 1977.

[12] S. S. Rao, Engineering Optimization: Theory and Practice, $3^{\text {rd }}$ ed. New York, Wiley, 1996

[13]H. C. Gea, "Topology Optimization: A new microstructure-based design domain method," Computers and Structures, vol. 61, no.5, pp.781788, 1996

[14]G. Kiziltas "Dielectric material optimization of filters and antennas using SIMP", Ph.D. Thesis in Mechanical Engineering, Ann Arbor: The University of Michigan, 2003

[15]R. Hanson and K. Hiebert, "A sparse linear programming subprogram," Technical Report SAND81-0297, Sandia National Lab., 1981

[16] G. Kiziltas, D. Psychoudakis, J. L. Volakis, and N. Kikuchi, "Topology Design Optimization of Dielectric Substrates for Bandwidth Improvement of a Patch Antenna," IEEE Transactions on Antennas and Propagation, Special Issue on Metamaterials, vol.51, no.10, pp. 2732-2743, October 2003

[17] Y. Koh, J.W. Halloran, A. M. Knapp, J. Volakis, G. Kiziltas, and D. Psychoudakis, "Textured dielectric meta-materials for miniaturized antennas: fabrication of dielectric LTCC-polymer by thermoplastic green machining", 105th Annual Meeting \& Exposition of The American Ceramic Society, Nashville, TN, April 2003 